\title{
CARACTERIZAÇÃO FILOGENÉTICA MOLECULAR E RESISTÊNCIA ANTIMICROBIANA DE Escherichia coli ISOLADAS DE CAPRINOS NEONATOS COM DIARREIA
}

\author{
MOLECULAR-PHYLOGENETIC CHARACTERIZATION AND \\ ANTIMICROBIAL RESISTANCE OF Escherichia coli ISOLATED FROM \\ GOATS WITH DIARRHEA
}

\author{
Rodrigo Almeida Guimarães ${ }^{1}$ \\ Domingos Fernandes Lugo Neto ${ }^{1}$ \\ Mauro de Mesquita Souza Saraiva ${ }^{1}$ \\ Ricardo Pereira Lima² \\ Mercia Rodrigues Barros ${ }^{3}$ \\ Mateus Matiuzzi da Costa ${ }^{4}$ \\ Celso Bruno Oliveira ${ }^{5}$ \\ Danilo Tancler Stipp ${ }^{2^{*}}$
}

\begin{abstract}
${ }^{1}$ Universidade Federal da Paraíba, Centro de Ciências Agrárias, Programa de Pós-Graduação em Ciência Animal, Areia, PB, Brasil.

'Universidade Federal da Paraíba, Centro de Ciências Agrárias, Departamento de Ciências Veterinárias, Areia, PB, Brasil.

3Universidade Federal Rural de Pernambuco, Departamento de Medicina Veterinária, Recife, PE, Brasil. ${ }^{4}$ Universidade Federal do Vale do São Francisco, Colegiado Acadêmico de Zootecnia, Petrolina, PE, Brasil. 5Universidade Federal da Paraíba, Centro de Ciências Agrárias, Departamento de Zootecnia, Areia, PB, Brasil. *Autor para correspondência - stipp@cca.ufpb.br
\end{abstract}

\section{Resumo}

A diarreia neonatal determina alterações significativas na conversão alimentar, resultando na queda da produtividade de caprinos. A resistência antimicrobiana em bactérias caracteriza-se como importante problema de saúde pública, assim, a Escherichia coli pode ser caracterizada como importante patógeno por expressar mecanismos de virulência responsáveis por significativas afecções clínicas em seres humanos e animais. O presente estudo avaliou a presença de E. coli em 117 amostras fecais de caprinos e analisou a resistência antimicrobiana dos isolados. Colônias sugestivas foram submetidas a testes bioquímicos, seguido de determinação do grupo genotípico e análise filogenética; posteriormente foram submetidas à prova de suscetibilidade a antimicrobianos. Foram identificados isolados de E. coli, Salmonella spp, Shigella sonnei e Enterobacter aerogenes. Isolados de E. coli foram classificados filogeneticamente em B2 (9/39), D (19/39), B1 (7/39) e A (4/29). Os fatores de virulência identificados foram K99 (04/39) e Stx (02/39). À prova de suscetibilidade antimicrobiana, os isolados foram sensíveis a Cloranfenicol, Estreptomicina, Amoxicilina e Ciprofloxacina, sendo todos resistentes à Lincomicina, Vancomicina e Penicilina. Os resultados reforçam a necessidade de estabelecimento de protocolos criteriosos para o uso de antimicrobianos, medida fundamental para o aprimoramento da sanidade dos rebanhos caprinos brasileiros.

Palavras-chave: antibióticos; bactérias; caprinos; E. Coli.

\section{Abstract}

Neonatal diarrhea determines significant changes in feed conversion, causing productivity loss in 
caprine herds. The antimicrobial resistance in bacteria is characterized as an important public health issue; therefore, Escherichia coli may be characterized as an important pathogen due to expressing virulence mechanisms responsible for significant clinical conditions in humans and animals. The present study evaluated the presence of E. coli among 117 caprine fecal samples and analyzed the isolates for antimicrobial resistance. Suggestive colonies were submitted to biochemical screening followed by genotypic group determination and phylogenetic analysis; further, the samples were submitted to antimicrobials susceptibility test. E. coli, Salmonella spp, Shigella sonnei and Enterobacter aerogenes were identified. E. coli isolates were phylogenetically classified as B2 (9/39), D (19/39), B1 (7/39) e A (4/29) groups. The analysis of the isolates also revealed the presence of K99 $(04 / 39)$ and Stx (02/39) virulence factors. Antimicrobial susceptibility test revealed sensitive isolates to Chloramphenicol, Streptomycin, Amoxicillin and Ciprofloxacin, being all resistant to Lincomycin, Vancomycin and Penicillin. The results support the need of establishing restricted protocols for antimicrobial use, a fundamental procedure for health improvement in Brazilian caprine herds.

Keywords: antibiotics; bacteria; caprine; E. Coli.

Enviado em: 18 janeiro 2015

Aceito em: 20 agosto de 2015

\section{Introdução}

As bactérias da família Enterobacteriaceae estão amplamente distribuídas na natureza, encontradas no solo, água, vegetais e no trato intestinal de seres humanos e animais. Os membros desta família são agrupados, arbitrariamente, em três categorias, sendo classificados como: patógenos principais determinantes de doenças entéricas e sistêmicas; patógenos oportunistas, que ocasionalmente determinam doença clínica além do trato alimentar; e não-patógenos.

Além da virulência, a resistência a antimicrobianos em cepas de Escherichia coli tem sido motivo de grande preocupação para a saúde pública, principalmente diante da evidência de que antibióticos exercem uma pressão seletiva tanto em bactérias patogênicas quanto nas comensais da microbiota. Desta forma, o aumento do uso de agentes antimicrobianos na clínica de pequenos animais, particularmente os de amplo espectro, tais como fluorquinolonas, cefalosporinas, e penicilinas associadas ao ácido clavulâmico têm agravado o problema da resistência microbiana. A emergência de fenótipos de resistência de relevância clínica como, por exemplo, as betalactamases de espectro estendido (ESBL) e a multirresistência em cepas de $E$. coli de origem animal ilustram bem a gravidade do problema ${ }^{(1)}$. Os antimicrobianos, quando usados como promotores de crescimento na alimentação animal, podem exercer forte pressão seletiva sobre os patógenos e a microbiota saprofítica, principalmente quando utilizados abusivamente, podendo resultar no aparecimento de resistência quer seja na microbiota saprofítica e/ou patogênica, em dependência da codificação de genes para resistência antimicrobiana pela ação de plasmídeos e transposons. A terapia antimicrobiana é comumente utilizada para controlar e prevenir infecções, mas esta prática pode selecionar bactérias resistentes. A ingestão de alimentos contendo resíduos de fármacos pode ocasionar resistência bacteriana aos antimicrobianos utilizados rotineiramente na terapêutica humana, dificultando o tratamento de enfermidades infecciosas humanas ${ }^{(2)}$. A resistência a drogas pode ser carreada por plasmídeos, bem como mutações cromossômicas que ocorrem espontaneamente ${ }^{(3)}$. Resistência a multidrogas têm sido descrita em humanos e suínos em isolados de E. coli. Bactérias formadoras de biofilmes são mais resistentes a drogas antimicrobianas, como foi determinado em outros patógenos veterinários, como Pseudomonas aeruginosa, Aeromonas hydrophila e Staphylococcus aureus ${ }^{(1)}$.

A origem evolutiva de E. coli, especialmente de isolados extraintestinais, tem sido estudada pela análise da presença de fatores de virulência em relação à organização populacional desta bactéria em diferentes grupos, a qual é definida por métodos filogenéticos ${ }^{(4)}$. Nas análises filogenéticas, temse verificado que cepas podem ser classificadas em quatro grupos principais conhecidos para esta espécie bacteriana, sendo: A, B1, B2 e $\mathrm{D}^{(5)}$. As cepas virulentas geralmente classificam-se no grupo 
B2, porém algumas são classificadas no grupo D. Por outro lado, as cepas comensais pertencem aos grupos $\mathrm{A}$ e $\mathrm{B} 1^{(6-8)}$. Cepas extraintestinais patogênicas e comensais de $E$. coli diferem de acordo com os fatores de virulência, expressos por genes geralmente agrupados em ilhas de patogenicidade, proporcionando um mecanismo de transferência horizontal coordenada desses genes de virulência ${ }^{(9)}$. Três candidatos a marcadores de patogenicidade têm sido estudados: chuA, um gene necessário para o heme-transporte em E. coli enterohemorrágica $(0157: \mathrm{H} 7)^{(10,11)}$; yjaA, um gene inicialmente identificado no genoma de $E$ coli $\mathrm{K} 12$, cuja função ainda é desconhecida ${ }^{(12)}$ e um fragmento de DNA designado TSPE4.C2(11). Cepas toxigênicas de E. coli causam diarreia aquosa profusa e/ou lesões vasculares sistêmicas devido à liberação de enterotoxinas como as toxinas termo-lábil (LT), termoestável (Sta e Stb) e Shiga toxina (Stx2e) ${ }^{(13)}$.

As fímbrias, também consideradas fatores de virulência, permitem a aderência dessas bactérias a receptores específicos localizados na superfície de enterócitos. Cinco diferentes tipos principais de fímbrias foram descritos em isolados de E. coli toxigênicas (ETEC) de origem suína: K88, K99, 987P, F18 e F41 ${ }^{(14)}$. A partir da aderência, essas bactérias colonizam a superfície celular e lá secretam as toxinas envolvidas no processo de diarreia. Após a adesão, E. coli enterotoxigênicas produzem toxinas (ST e LT) que induzem a hipersecreção pelas células do intestino ou que interferem na síntese proteica das células (Stx2e) acarretando diarreia nos animais ${ }^{(15-17)}$.

O presente estudo teve como objetivo identificar cepas de E. coli, a partir de isolados obtidos de fezes de caprinos com diarreia, determinar o perfil de resistência das cepas isoladas frente a diferentes antimicrobianos e identificar os fatores de virulência e sua classificação filogenética.

\section{Material e Métodos}

No período de março de 2013 a maio de 2014 foram coletadas 117 amostras de fezes diarreicas de caprinos em 24 propriedades, no estado da Paraíba, com até 90 dias de idade. As amostras foram armazenadas em frascos universais de coleta estéreis e sob refrigeração por até 15 dias.

A identificação bacteriana foi realizada por meio de etapa prévia de enriquecimento em caldo Brain Heart Infusion (BHI) e, posteriormente, semeadas em meio Levine com incubação por 24 horas a $37,5^{\circ} \mathrm{C}$. As colônias sugestivas de enterobactérias foram submetidas à análise bioquímica por meio de inoculação em meio TSI, Citrato, SIM, VM e VP, com leitura após 24 horas e análise de acordo com o manual Bergey. As colônias sugestivas foram estocadas, mantidas em caldo Triple Sugar Iron (TSI) e, posteriormente, transferidas para BHI glicerinado, sendo mantidas sob à temperatura de -20 ${ }^{\circ} \mathrm{C}$ até o seu uso.

Os isolados foram enriquecidos em caldo BHI e novamente semeados sem Agar MacConkey, sendo incubados por 24 horas a $37^{\circ} \mathrm{C}$. Colônias isoladas foram coletadas e ressuspendidas em microtubo contendo $500 \mu \mathrm{L}$ de água estéril ultrapura. As suspensões foram aquecidas a $100{ }^{\circ} \mathrm{C}$ por 10 minutos e, em seguida, mantidas em gelo por 10 minutos e centrifugadas a 12.000 RPM por 3 minutos, sendo retirado ao final do processo o sobrenadante com o DNA bacteriano.

Para a realização da técnica de multiplex-PCR (mPCR) foi preparada solução "mastermix" composta de: $15 \mu \mathrm{L}$ de $\mathrm{H}_{2} \mathrm{O}$ milli-Q, $2,5 \mu \mathrm{L}$ de tampão, $1 \mu \mathrm{L} \mathrm{MgCl}_{2}, 1 \mu \mathrm{L}$ dNTPs, $1 \mu \mathrm{L}$ de cada primer (Tab 01) e $0,5 \mu \mathrm{L}$ Taq polimerase. Foram utilizados $22 \mu \mathrm{L}$ da solução "mastermix" acrescidos de $3 \mu \mathrm{L}$ de água estéril ultrapura como controle negativo e $3 \mu \mathrm{L}$ de cada amostra de DNA. A amplificação dos genes ChuA (279pb) e YjaA (211pb) consistiu de desnaturação a $94{ }^{\circ} \mathrm{C}$ por 4 min.; seguido por 30 ciclos de $94{ }^{\circ} \mathrm{C}$ por 30 s, $55^{\circ} \mathrm{C}$ por 30 s e $72{ }^{\circ} \mathrm{C}$ por 30 s; com extensão final à $72{ }^{\circ} \mathrm{C}$ por 7 min. A amplificação do gene TspE4C2 (152pb) consistiu de desnaturação a $94{ }^{\circ} \mathrm{C}$ por 3 min.; seguido por 35 ciclos de 94 ${ }^{\circ} \mathrm{C}$ por 3 min., $55^{\circ} \mathrm{C}$ por $1 \mathrm{~min}$. e $72{ }^{\circ} \mathrm{C}$ por $45 \mathrm{~s}$; com extensão final à $72{ }^{\circ} \mathrm{C}$ por $7 \mathrm{~min}$. A amplificação dos genes de virulência K99 (543pb), Stx (758pb), K88 (855pb), Sta (158pb), StbP (113pb) e LT (272pb) consistiu de 35 ciclos de $94{ }^{\circ} \mathrm{C}$ por 30 s, $55^{\circ} \mathrm{C}$ por 30 s e $72{ }^{\circ} \mathrm{C}$ por 90 s; com extensão final à $72{ }^{\circ} \mathrm{C}$ por $10 \mathrm{~min}$. O gel foi visualizado sob luz ultra violeta e fotografado.

Para o teste de sensibilidade a antimicrobianos, foi utilizada a técnica de difusão em ágar, segundo as 
recomendações de Woods e Washington ${ }^{(18)}$. A partir das amostras de E. coli isoladas em ágar triptona de soja por $24 \mathrm{~h}$ a $37^{\circ} \mathrm{C}$, foi utilizada uma solução salina autoclavada e diluídas as colônias de bactérias até se atingir o equivalente à escala 0,5 MacFarland e inoculadas em ágar Muller-Hinton. As placas foram incubadas por $18 \mathrm{~h}$ a $37^{\circ} \mathrm{C}$. Foram utilizados discos de Amoxicilina e Ácido Clavulâmico (i30), Ampicilina (i10), Cefalexina (i30), Ciprofloxacina(i5), Cloranfenicol (i30), Estreptomicina (i300), Gentamicina (i10), Lincomicona (i2), Penicilina G (i10), Sulfametoxazoletrimetopima (i25), Tetraciclina (i30), Vancomicina (i30). Após a incubação, os halos de inibição foram medidos e os padrões de susceptibilidade foram determinados segundo o Manual Clinical and Laboratory Standards Institute (CSLI, 2007) ${ }^{(19)}$.

\section{Resultados}

As amostras foram submetidas às análises bioquímicas e identificadas resultando em 47\% (55/117) de isolados indiferenciados, 33\% (39/117) de isolados de E. coli, 10\% (12/117) de Shigella sonnei, 6,83\% (8/117) de Enterobacter aerogenes, 0,85\%(1/117) de Salmonela spp., 0,85\% (1/117) de Providencia spp. e $0,85 \%$ (1/117) de Klebsiella pneumoniae.

A escolha dos 12 agentes antimicrobianos para verificar as suscetibilidades entre os isolados clínicos foi baseada em protocolos sugeridos pelo Clinical and Laboratory Standards Institute (CLSI) ${ }^{(19)}$ para Enterobactérias Gram negativas. Os antibióticos testados são de uso frequente na clínica veterinária de animais de produção.

A prova de sensibilidade a antimicrobianos demonstrou que todas as amostras foram resistentes à Lincomicina, Vancomicina e Penicilina, seguido de 38,46\% (15/39) à Tetracicilina, 35,89\% (14/39) à Cefalexina e 33,33\% (13/39) à Sulfametoxazol e Trimetopima. A sensibilidade bacteriana encontrada nas amostras analisadas foi de 92,30\% (36/39) para Estreptomicina, Amoxicilina e Ácido Clavulânico, e Cloranfenicol.

Com relação à multirresistência a drogas (MDR), caracterizada como a resistência a três ou mais agentes antimicrobianos, algumas amostras obtiveram $100 \%$ de resistência. $O$ fenótipo mais frequente de MDR foi o de resistência à Penicilina,Vancomicina e Lincomicina de 100\% das cepas (Fig. 1).

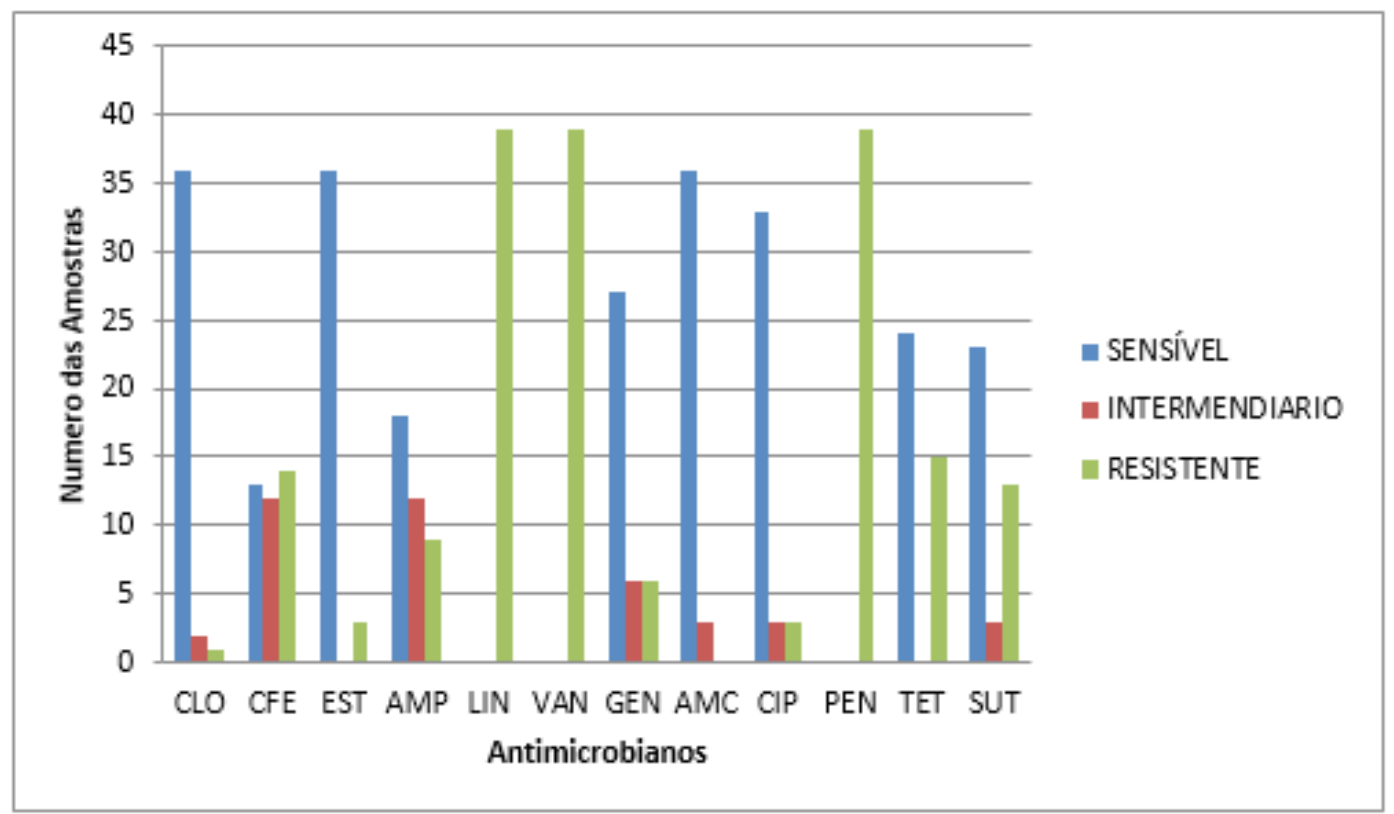

Figura 1:Representação da resistência, sensibilidade intermediária e sensibilidade entre as cepas isoladas. 
Os isolados de E. coli foram caracterizados genotipicamente, sendo 10,2\% (4/39) pertencentes ao grupo A, 17,9\% (7/39) ao grupo B1, 23,07\% (9/39) ao grupo B2 e 48,71\% (19/39) ao grupo D. Considerando que cepas virulentas são geralmente classificadas nos grupos B2 e D, um total de $71,78 \%$ (28/39) das cepas caracterizadas são possíveis de serem patogênicas. Seis (15\%) amostras analisadas foram positivas para um ou mais fatores de virulência, sendo quatro $(10,2 \%)$ positivas para K99 e duas $(5,12 \%)$ para Stx, indicando a presença de possível potencial patogênico nas amostras ${ }^{(20)}$.

\section{Discussão}

A caracterização de E. coli quanto ao padrão de resistência a antibióticos e a identificação do padrão de virulência são informações fundamentais para a caracterização de isolados clínicos, tanto de animais, como da água e do ambiente; além de possibilitarem discriminar o potencial patogênico dessas bactérias e a identificação de clones patogênicos emergentes ${ }^{(21)}$. Bactérias estão amplamente disseminadas em diferentes espécies de hospedeiros e também no meio ambiente. Além disso, podem apresentar multirresistência a antimicrobianos comumente utilizados na produção ${ }^{(2,23)}$. A lincomicina é um dos antimicrobianos autorizados no Brasil para uso na avicultura, como aditivo na alimentação. O uso de antibióticos nas medicinas veterinária e humana é considerado um fator importante para promover a seleção e disseminação de microrganismos resistentes ${ }^{(24)}$. Neste trabalho, verificou-se também que, independentemente do tipo de criação, os isolados de E. coli apresentaram resistência de 100,0\% para lincomicina. A produção da enzima Beta-lactamase é o mais comum mecanismo de produção de resistência frente a antibióticos beta-lactâmicos em bactérias Gram negativas. $\mathrm{O}$ uso contínuo de antibióticos de amplo espectro induz a produção destas enzimas ${ }^{(25)}$.

Dentre os isolados analisados, todos apresentaram resistência há pelo menos três dos agentes antimicrobianos testados e $75 \%$ apresentaram resistência a mais de três antimicrobianos. A multirresistência tem sido citada por diversos autores ${ }^{(21,26)}$, apresentando níveis mais elevados em cepas isoladas de suínos quando comparada a bovinos e ovelhas, fato que se deve à pressão seletiva causada pelo uso indiscriminado de antimicrobianos, provavelmente na ração animal como promotor de crescimento ${ }^{(27)}$. Esta resistência é importante, podendo ser transmitida aos humanos, tanto através da colonização direta do intestino por cepas de E. coli animal quanto pela transmissão de genes de resistência a bactérias residentes no intestino humano.

O maior índice de sensibilidade foi observado para Amoxicilina, Ácido Clavulânico, Estreptomicina e Cloranfenicol, em 92,30\% dos isolados; seguido por Ciprofloxacina em 84,61\% dos isolados. Em estudo realizado em carcaças suínas, obteve-se porcentagem semelhante à do presente estudo em relação à sensibilidade dos isolados à Ciprofloxacina, com $88,2 \%{ }^{(23)}$, mas com diferença de resultados em relação à Nitrofurantoína, em que apenas 17,6\% dos isolados foram sensíveis. A divergência observada pode ser atribuída à variabilidade das estirpes e ao crescente aumento da resistência antimicrobiana entre as enterobactérias ${ }^{(28)}$. Aos antimicrobianos Sulfametoxazol e Trimetoprim, $87,5 \%$ dos isolados foram sensíveis, resultado semelhante ao descrito por Varga et al. ${ }^{(29)}$, que verificaram $93,6 \%$ dos isolados de E. coli, de suínos saudáveis, sensíveis ao mesmo. A maior resistência dos isolados foi observada para tetraciclina com 93,7\%. Isto não é surpreendente, uma vez que a tetraciclina é utilizada há muitos anos como promotor de crescimento para animais.

Apesar de sua proibição de uso em animais de produção, um isolado $(2,56 \%)$ foi resistente ao Cloranfenicol, e dois isolados $(5,12 \%)$ com sensibilidade intermediária, indicando seu possível uso em animais de produção. A identificação de resistência para Cloranfenicol indica um sério problema à saúde pública. Desta maneira, monitorar a resistência de bactérias isoladas de animais a diferentes grupos de antibióticos torna-se um fator determinante para eleição e êxito do tratamento, bem como eliminar, através do uso de antibióticos específicos, a presença de linhagens carreadoras de genes relacionados à patogenicidade de $E$. coli.

A análise filogenética determinou maior proporção de isolados classificados nos grupos B2 e D, sendo estes grupos os de maior virulência e com possibilidades de serem patogênicos. Estes resultados 
são semelhantes aos descritos por Bingen et al. ${ }^{(30)}$ em humanos. Estudos demonstraram que as mais importantes cepas de E. coli encontradas pertenciam aos grupos B1 e D, diferente de dados obtidos em estudos com humanos, os quais observaram maior prevalência de isolados dos grupos A e B2. Em outro estudo, foi descrita uma maior incidência de isolados do grupo D em amostras aviárias $^{(31)}$. Em amostras de origem humana, são comumente detectados isolados do grupo B2(32). Essas diferenças podem estar relacionadas com a alta sensibilidade a antimicrobianos e à maior ou menor virulência. Em comparação, cepas isoladas de animais são mais resistentes a antimicrobianos. Algumas variações podem estar associadas a regiões geográficas e/ou características individuais das espécies de hospedeiros.

Estudos sugerem que os isolados de E. coli podem ter uma origem múltipla, uma de linhagem patogênica e outra de linhagem não patogênica possivelmente aperfeiçoada pela aquisição horizontal de genes virulentos. Silveira et al. ${ }^{(33)}$ e Baccaro et al ${ }^{(20)}$ pesquisaram genes de toxinas (StaP, Stb e LT) e fímbrias (K88, K99, 987P, F41) isoladas de suínos diarreicos e identificaram, respectivamente, 27,3\% e $24,3 \%$ das amostras com fatores de virulência. No presente estudo, as frequências de detecção dos genes K99 foi de 10,25\% e de 5,12\% para o gene Stx, diferentemente do que foi descrito por aqueles autores.

Fairbrother et al. ${ }^{(16)}$ analisaram, por meio da PCR, isolados de E. coli de suínos com diarreia e constataram que em $75 \%$ e $65,5 \%$ dos casos, respectivamente, as amostras apresentaram resultado positivo para pelo menos um dos genes fimbriais (F18, F41, K88 e K99) ou toxinas (Sta, Stb, LT e Stx2e) pesquisados. No entanto, a análise para o gene Stx2 não foi incluída no estudo de Fairbrother et al. ${ }^{(16)}$ e as amostras analisadas foram positivas para apenas um dos genes testados, isto é, amostras positivas para o gene Stx não foram positivas para nenhum outro gene testado, e amostras positivas para o gene fimbrial K99 também não foram positivas a nenhum outro gene.

\section{Conclusão}

Os isolados de E. coli foram identificados como pertencentes a grupos filogenéticos de elevada virulência (B2 e D), sendo observados fatores de virulência (K99 e Stx) que indicam potencial patogênico significativo das amostras, o que apresenta uma provável relação como determinante da diarreia nos rebanhos investigados.

Foi observada significativa resistência antimicrobiana entre algumas drogas testadas, implicando uma emergente necessidade de controle das drogas disponíveis para o uso em Medicina Veterinária e também a necessidade de descoberta de novas drogas que não possuam elevado índice de resistência. Os resultados reforçam a necessidade de estabelecimento de protocolos criteriosos para o uso de antimicrobianos para se limitar esta resistência.

A utilização de métodos eficazes de diagnóstico de microrganismos envolvidos nas infecções constitui uma importante etapa do processo de vigilância quanto à identificação de cepas bacterianas resistentes a antimicrobianos. Além disso, atualizações constantes por meio de publicações de novos estudos sobre o estado de resistência aos antimicrobianos, especialmente os mais utilizados na medicina veterinária, são fundamentais para o aprimoramento da sanidade dos rebanhos caprinos brasileiros.

\section{Referências}

1 - Allen H, Donato J, Wang, Cloud-Hansen K, Davies J, Handelsman J. Call of the wild: antibiotic resistance genes in natural environments. Nature Reviews Microbiology, 2010; 8: 251-259.

2 - Mantilla S, Franco R, Oliveira L, Santos E, Gouvêa E. Resistência antimicrobiana de bactérias do gênero Listeria spp. isoladas de carne moída bovina. Brazilian Journal of Veterinary Research and Animal Science, 2008, 45(2): 116-121.

3 - Lázár V, Nagy I, Spohn R, Csörg B, Györkei A, Nyerges A, Horváth B, Vörös A, Busa-Fekete R, Hrtyan 
M, Bogos B, Méhi O, Fekete G, Szappanos B, Kégl B, Papp B, Pál C. Genome-wide analysis captures the determinants of the antibiotic cross-resistance interaction network. Nature Communications. 2014; 5: 4352.

4 - Johnson JR, Phylogenetic distribution of extraintestinal virulence associated traits in Escherichia coli. The Journal of Infectious Diseases, 2001; 183: 78-88.

5 - Clermont, O; Bonacorsi, S; Bingen, E: Rapid and Simple Determination of the Escherichia coli Phylogenetic Group. Applied Environment Microbiology, 2000; 66:4555- 4558.

6 - Herzer, PJ. Phylogenetic distribution of branched RNS-linked multicopy single-stranded DNA among natural isolates of Escherichia coli. Journal of Bacteriology, 1990; 172: 6175-6181.

7 - Lecointre G, Escherichia coli molecular phylogeny using the incongruence length difference test. Molecular Biology and Evolution, 1998, 15(12): 1685-1695.

8 -Sabaté M, Moreno E, Pérez T, Andreu A, Prats G: Pathogenicityislandmarkers in commensalanduropathogenic Escherichia coli isolates. Clinic Microbiology Infection, 2006; 12:880-886.

9 - Sherley M, David M. Gordon, and Peter J. Collignon. Evolution of multi-resistance plasmids in Australian clinical isolates of Escherichia coli. Microbiology, 2004; 150(5): 1539-1546.

10 - Mills M, Payne S. Genetics and regulation of haem iron transport in Shigelladysenteriae and detection of an analogous system in Escherichia coli O157:H7. Journal of Bacteriology, 1995, 177(11): 3004-3009.

11 - Bonacorsi S, Clermont O, Tinsley C, Le Gall I, Beaudoin J, Elion J, Nassif X, Bingen E. Identification of regions of the Escherichia coli chromosome specific for neonatal meningitis-associated strains. Infectum Immunologic, 2000; 68: 2096-2101.

12 - Blattner, F. R. Plunkett G, Bloch C, Perna N, Burland V, Riley M, Collado-Vides J, Glasner J, Rode C, Mayhew G, Gregor J, Davis N, Kirkpatrick H, Goeden M, Rose D, Mau B, Shao Y. The complete genome sequence of Escherichia coli K-12. Science, 1997; 277(5331): 1453-1461.

13 - Francis, D. Enterotoxigenic Escherichia coli infection in pigs and its diagnosis. Journal of Swine Health Production, 2002; 10(4): 171-175.

14 - Wilson, R, Francis, D Fimbriae and enterotoxins associated with Escherichia coli serogroups isolated from pigs with colibacillosis. American Journal of Veterinary Research, 1986, 47(2): 213-217.

15 - Holland I, Kenny B, Blight M. Haemolysin secretion from E. coli. Biochimie, 1990; 72(2): 131-141.

16 - Fairbrother J, Gyles C. Escherichia coli infections. Diseases of Swine, 2006; 9: 639-674.

17 - Fairbrother J, Nadeau E, Gyles C. Escherichia coli in postweaning diarrhea in pigs: an update on bacterial types, pathogenesis, and prevention strategies. Animal Health Research Reviews, 2005; 6(1): 17-39.

18 - Woods GL. Antibacterial susceptibility tests: dilution and disk diffusion methods. In: Murray P. Manual of Clinical Microbiology. 6th ed. Washington: ASM Press, 1995. p.1327-1341. English.

19 - CLSI. Methods for Dilution Antimicrobial Susceptibility Tests for Bacteria That Grow Aerobically: Approved Standard - Sixth Edition, Pennsylvania, 19087-1898, USA, 2003

20 - Baccaro M., Moreno A, Corrêa A, Ferreira A, Calderano F. Resistência antimicrobiana de amostras de Escherichia coli isoladas de fezes de leitões com diarréia. Arquivos do Instituto Biológico, 2002; 69: 15-18.

21 - Barros M, Silveira W, Araújo J, Costa E, Oliveira A, Santos A. Resistência antimicrobiana e perilplasmidial de Escherichia coli isolada de frangos de corte e poedeiras comerciais no Estado de Pernambuco. Pesquisa Veterinária Brasileira, 2012; 32(5): 405-410.

22 - Parveen S, Lukasik J, Scott T, Tamplin M, Portier K, Sheperd S. Braun K, Farrah S. Geographical variation in antibiotic resistance profiles of Escherichia coli isolated from swine, poultry, beef and dairy cattle farm water retention ponds in Florida. Journal of Applied Microbiology, 2006, 100(1): 50-57.

23 - Paterson D. Resistance in gram-negative bacteria: Enterobacteriaceae. The American Journal of Medicine, 2006, 119(6): S20-S28.

24 - White D, Zhao S, Sudler R, Ayers S, Friedman S, Chen S, McDermott P, McDermott S, Wagner D, Meng J. The isolation of antibiotic-resistant salmonella from retail ground meats. The New England Journal of Medicine, 2001; 345(16): 1147-1154.

25 - Dolejska M, Cizek A, Literak I. High prevalence of antimicrobial-resistant genes and integrons in Escherichia coli isolates from Black-headed Gulls in the Czech Republic. Journal of Applied Microbiology, 
2007; 103(1): 11-19.

26 - Guerra B, Junker E, Schroeter A, Malorny B, Lehmann S, Helmuth R. Phenotypic and genotypic characterization of antimicrobial resistance in German Escherichia coli isolates from cattle, swine and poultry. Journal of Antimicrobial Chemotherapy, 2003; 52: 489-492.

27 - Enne V, Cassar C, Sprigings K, Woodward M, Bennett P. A high prevalence of antimicrobial resistant Escherichia coli isolated from pigs and a low prevalence of antimicrobial resistant $E$. coli from cattle and sheep in Great Britain at slaughter. Federation of European Microbiological Societies - Microbiology Letters, 2008; 278: 193-199.

28 - Costa M, Silva M, Spricigo D, Witt N, Marchioro S, Kolling L, Vargas A. Caracterização epidemiológica e perfil de resistência aos antimicrobianos de Escherichia coli isoladas de criatórios suínos do sul do Brasil. Pesquisa Veterinária Brasileira, 2006; 26: 5-8.

29 - Varga C, Raji'c A, McFall M, Avery B, Reid-Smith R, Deckert A, Checkley S, McEwen S. Antimicrobial resistance in generic Escherichia coli isolated from swine fecal samples in 90 Alberta finishing farms. The Canadian Journal of Veterinary Research, 2008; 72: 175-180.

30 - Bingen E, Picard B, Brahimi N, Mathy S, Desjardins P, Elion J, Denamur E. Phylogenetic analysis of Escherichia coli strains causing neonatal meningitis suggests horizontal gene transfer from a predominant pool of highly virulent B2 group strains. Journal of Infectious Diseases, 1998; 177(3): 642-650.

31 - Campos T, Nakazato G, Stehling E, Brocchi M, Silveira W. Estudo clonal de Escherichia coli aviário por análise de seqüências de DNA conservadas do gene fliC. Pesquisa Veterinária Brasileira, 2008; 28(10): 508-514.

32 - Maynard C, Bekal S, Sanschagrin F, Levesque RC, Brousseau R, Masson L, Larivière S, Harel J. Heterogeneity among virulence and antimicrobial resistance gene profiles of extraintestinal Escherichia coli isolates of animal and human origin. Journal of clinical microbiology, 2004; 42(12): 5444-5452.

33 - Silveira W, Ferreira A, Brocchi M, Maria de Hollanda L, Pestana de Castro AF, Tatsumi Yamada A, Lancellotti M. Biological characteristics and pathogenicity of avian Escherichia coli strains. Veterinary Microbiology, 2002; 85: 47-53. 